Natural and Applied Sciences International Journal (NASIJ) eISSN: 2788-4619 (online)

https://doi.org/10.47264/idea.nasij/2.1.5

Vol. 2, No. 1 (January-December 2021), 56-69

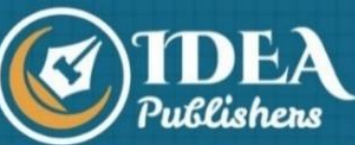

https://www.ideapublishers.org/index.php/nasij

Research Article

\title{
Magnetometer heading estimation through online calibration for land navigation applications
}

\author{
Muhammad Iqbal*1,2 $\mid$ Masood-ur-Rehman ${ }^{1} \mid$ Umar Iqbal Bhatti $^{1}$ | Najam Abbas Naqvi ${ }^{1,2}$ \\ 1. Department of Space Sciences, Institute of Space Technology, Islamabad, Pakistan. \\ 2. Space Education and GNSS Lab, National Center of GIS and Space Application, Institute of \\ Space Technology, Islamabad, Pakistan. \\ * Corresponding Author Email: m4muhammadiqbal@gmail.com
}

Published Online: December 16, 2021

\begin{abstract}
For land navigation applications, the integration of the magnetometer with the combination of MEMS-INS and the Global Navigation Satellite System (GNSS) give excellent results. During land navigation applications, the magnetometer's heading can also be used during the GNSS outages. The calibration of the magnetometer is indispensable to calculate its accurate heading. There exist several methods for magnetometer calibration. Some are offline and some are online calibration techniques. In this paper, a calibration method is proposed to estimate the magnetometer's parameters through online calibration in run time. In this method, the reference magnetic field is calculated from the World Magnetic Model (WMM-2020). Moreover, reference roll, pitch and heading are provided from some other sources such as GNSS, Attitude Heading Reference System (AHRS), or reference INS. For different roll and pitch sectors, calibration parameters are estimated and stored. These parameters are used for magnetometer online calibration during the field testing. Both the headings obtained by the online calibration and conventional lab calibrations are analysed. Furthermore, the heading estimated through the online calibration is autonomous and fast. Subsequently, there is no user involvement in this online calibration technique and no specific movements to the device are provided. The heading obtained by novel technique is as accurate as obtained by conventional offline lab calibration.
\end{abstract}

Keywords: GNSS, MEMS-INS, AHRS, MEMS, World Magnetic Model, magnetometer, navigation, navigation applications, calibration.

How to Cite: Iqbal, M., Rehman, M. U., Bhatti, U. I., \& Naqvi, N. A. (2021). Magnetometer heading estimation through online calibration for land navigation applications. Natural \& Applied Sciences International Journal (NASIJ), 2(1), 56-69. https://doi.org/10.47264/idea.nasij/2.1.5

Publisher's Note: IDEA PUBLISHERS (IDEA Journals Group) stands neutral regarding jurisdictional claims in the published maps and institutional affiliations.

Copyright: ( 2021 The Author(s), published by IDEA PUBLISHERS (IDEA Journals Group)

Licensing: This is an Open Access article published under the Creative Commons AttributionNonCommercial 4.0 International License (http://creativecommons.org/licenses/by-nc/4.0/) 


\section{Introduction}

The magnetometer is a widely available low-cost sensor that can be used during land navigation applications to provide heading information. The magnetometer estimates the heading by sensing the earth's magnetic field vectors. Nowadays, due to advancement in the MEMS technology, lightweight and low-cost heading and attitude reference systems are manufactured which are comprised of a magnetometer and an Inertial Measurement Unit. This system is used for numerous low-cost navigation applications. The advancement in the MEMS technology made it possible to manufacture cheap, lightweight and accurate MEMS Inertial Measurement Units that are embedded with electronics to serve as MEMS-INS. These INS are used in vast areas for medium or cheap navigation applications (Noureldin et al., 2013). They are also used today in a variety of applications due to their reliability and robustness (Chiang et al., 2003; Aggarwal et al., 2008).

MEMS-INS is mainly used for short-timed navigation applications (Caruso, 1997; 2000; D'Amato et al., 2021). The position and attitude errors increase unboundedly with time and similarly, the performance is degraded with time for longer time applications as their errors are not bounded (Zhou et al., 2021). More efforts are going on to increase their performance for long term navigation solutions. To keep the errors, inbound and in an acceptable level regular updates like velocity, position and attitude is required from an external source (Fan et al., 2014). Therefore, MEMS-INS stand alone cannot serve the best for navigation applications due to its accumulated errors (Zhou et al., 2021). For better outcomes and to obtain an optimized navigation solution, MEMS-INS has to be integrated with another reliable aided navigation system like GNSS (Gebre-Egziabher et al., 2006; Groves, 2013; Javed \&Tahir, 2021). The GNSS provides position, navigation and time information to the user at the globe and even in space (Wahdan et al., 2014). Therefore, the MEMS-INS is integrated with the GNSS during the integrated navigation applications, where their errors are compensated by the GNSS updates (Fan et al., 2014). Furthermore, the position and velocity updates from the GNSS are utilized to minimize and keep the MEMS-INS errors inbound (Kok \& Shön, 2016; Lee et al., 2020).

During the navigation of vehicles in an urban or mountainous area, the GNSS signals are degraded due to signal obstructions from local topography and buildings (Kaplan \& Hegarty, 1996; Mansoor et al., 2019). The signals may experience multipath, spoofing, jamming and blockage. As a result, position, navigation and time estimation cannot be obtained accurately by GNSS (Yongjian et al., 2021). In order to obtain position, navigation, time and velocity from GNSS, good visibility to the satellites are required (Ozevin, 2014). The blockage of signals may produce multipath phenomena and weakens satellite availability. Thus, navigation solutions may not be available and optimal integrated navigation solutions can't be attained. The errors that arose in MEMS-INS cannot be bounded due to GNSS signal outages (Lim et al., 2018). Therefore, accurate and continuous navigation in an urban environment cannot be guaranteed during MEMS-INS and GNSS integrated navigation applications during GNSS signal outages. Thus, MEMS-INS and GNSS integrated navigation system must be augmented with another reliable system to ensure continuous and accurate navigation solution during GNSS signal outages (Ilyas et al., 2012; Rabiain et al., 2013).

The magnetometer is also a low-cost sensor. It senses the earth's magnetic field and provides an absolute heading. Magnetometer output is usually corrupted by external magnetic fields other than the earth's magnetic field. Magnetometers errors can be classified into biases, scale 
factors, misalignments, hard and soft iron (Titterton et al., 2004). Calibration of the magnetometer is essential before its use. There exist many methods of magnetometer calibration. Hence, magnetometer is used in MEMS-INS, GNSS integration, which can provide accurate heading information (Skog \& Händel, 2006; Wahdan et al., 2014).

The prime motive of the study is to use the inexpensive navigation system for land navigation applications. An AHRS is the best solution for this purpose. The magnetometer portion of an AHRS needs to be calibrated first. As, if we have used the magnetometer without its calibration, then we cannot achieve the goal. Therefore, an easy and fast online calibration technique is used to estimate the magnetometer's parameters. By using the calibrated parameters, we can obtain an accurate heading. The rest of the paper is organized as: the section 2 is about the data and research methodology; section 3 is comprised of the field testing and the results of the study; and section 4 consists of discussion. Finally, section 5 consists of brief conclusions based on the findings of study.

\section{Data and method}

The intensity of the magnetic field due to the Earth is 0.5 to 0.6 gauss. Its components are always parallel to the earth's surface and always pointing towards the earth's magnetic north. Earth's magnetic north and true north do not coincide (Yang, et al. 2013). There is an angle between the earth's magnetic north and true north. This angle is named as declination angle or inclination angle which depends on the direction of measurements. The heading of any vehicle with respect to the magnetic north can be estimated by measuring the earth's true magnetic field components (Won et al., 2015).

Figure 1: Magnetic north, true north, magnetic heading and declination angle (Won et al., 2015)

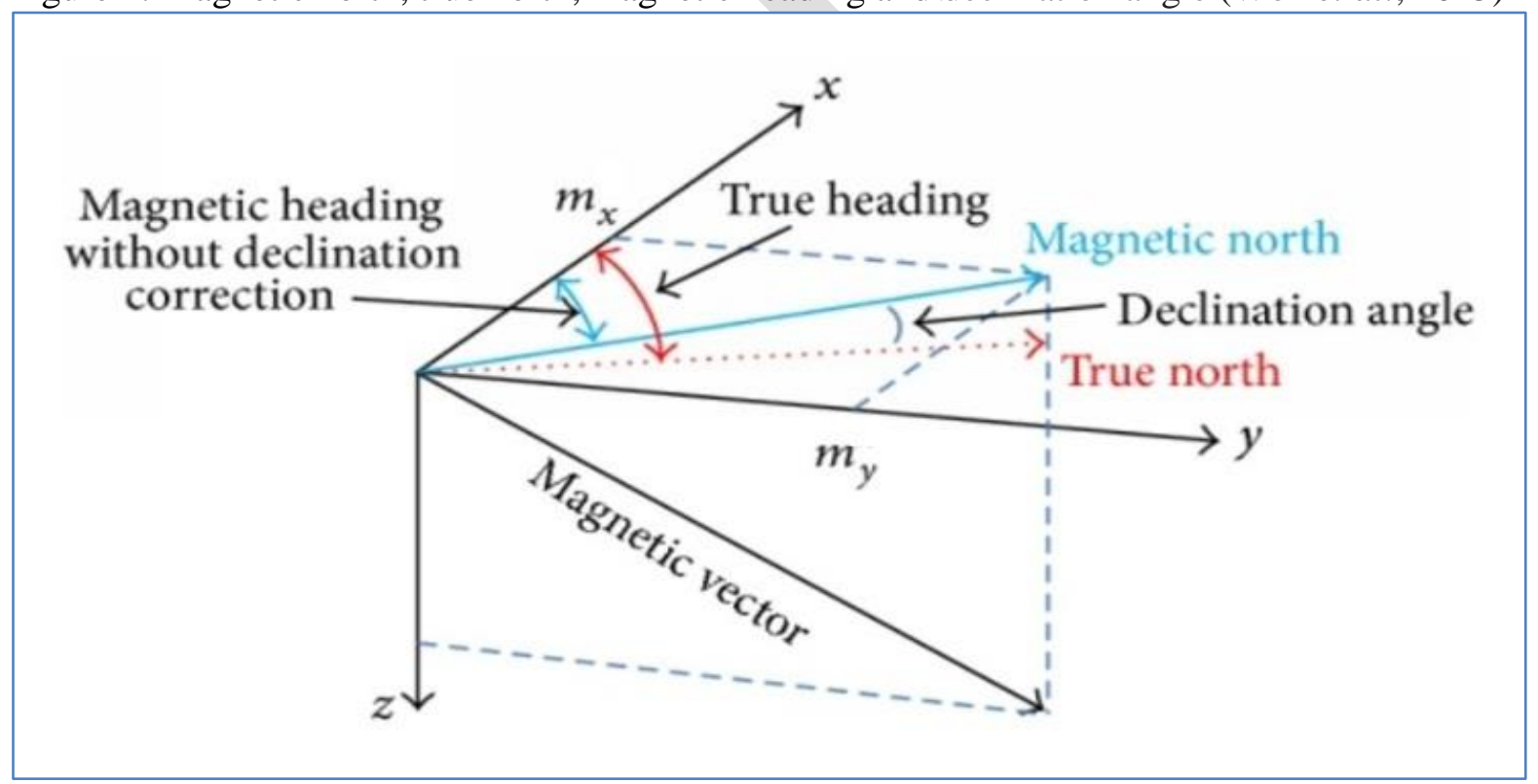

For estimation of heading with respect to geographic or true north, the declination angle is added for west direction values and is subtracted from east direction values. By using an updated magnetic field model, the declination angle at any place on earth can be easily estimated. By using the earth's magnetic field components and declination angle, the heading can be estimated by using the following equation. 


$$
\psi=\arctan .\left(\frac{M y}{M x}\right) \pm \text { Dec angle }
$$

Where, $M x$ and $M y$ represent the horizontal and vertical components of Earth's magnetic field, respectively.

\subsection{Main objective of the research}

The main objective of this research is to use the heading of low-cost magnetometer during land navigation applications. Therefore, the main task is to calibrate the magnetometer to find its accurate parameters like biases, scale factors, misalignments and magnetic distortions etc. True and accurate heading is finally computed from the magnetometer's accurately estimated parameters. Then, the calibrated heading is utilized during integrated land navigation applications.

\subsection{Magnetometer parameters}

The magnetometer raw readings are not precise and has some errors. These errors are required to be estimated and compensated before using the magnetometer readings. By performing magnetometer calibration, the different errors are estimated. The accurate, precise and calibrated readings of magnetometer are obtained by the compensation of these errors. Therefore, calibration technique of magnetometer is very important. An accurate, fast and autonomous calibration technique for precise readings estimation is essential. Therefore, a novel online calibration technique is utilized to estimate the calibrated parameters of the magnetometer. The precise heading is attained by the magnetometer's calibrated parameters. When accurate heading is utilized during the integrated navigation applications the best navigation results are achieved.

Some important parameters of magnetometer include a) Biases/off-set errors; b) Scale factors; c) Misalignment errors; d) Hard iron effects of magnetometer; and e) Soft iron effects of magnetometer. First three errors are instrumentation errors whereas, the last two are magnetic deviation which depends on the surrounding magnetic field anomalies. Instrumentation errors are due to fabrication limitations. They can be considered as unique and constant for one specific triad of magnetometers.

The scale factor matrix $\mathrm{S}$ represents these as,

$\mathrm{S}=\operatorname{diag}\left(\begin{array}{lll}S_{x} & S_{y} & S_{z}\end{array}\right)$

Where, $S_{x}, S_{y} \& S_{z}$ are magnetometer's x, y and z axis scale factors.

The magnetometer sensor offset introduces a bias in the output. The biases can be modelled as,

$\mathrm{b}=\left[\begin{array}{lll}b_{\mathrm{x}} & b_{\mathrm{y}} & b_{\mathrm{z}}\end{array}\right]$

Where, $b_{x}, b_{y} \& b_{z}$ are magnetometer's $\mathrm{x}, \mathrm{y}$ and $\mathrm{z}$ channels offsets.

Ideally, the magnetometer axes are perfectly aligned, and they are orthogonal with each other. Therefore, misalignment errors are considered negligible and are not calculated. 


\subsection{Magnetometer calibration}

Magnetometer output can be expressed by the following equations. The reference vector of a magnetic field in the device frame can be expressed by the following equations:

$$
\begin{aligned}
& M_{x}^{\prime}=b_{x}+S_{x} M_{x} \\
& M_{y}^{\prime}=b_{y}+S_{y} M_{y} \\
& M_{z}^{\prime}=b_{z}+S_{z} M_{z}
\end{aligned}
$$

Where,

$M_{x}^{\prime}, M_{y}^{\prime}$ and $M_{z}^{\prime}$ are raw/un-calibrated magnetometer output.

$M_{x}, M_{y}$ and $M_{z}$ are magnetic field vectors obtained from earth magnetic model, transformed in body frame.

$b_{x}, b_{y}$ and $b_{z}$ are biases readings in the device frame.

$S_{x}, S_{y}$ and $S_{z}$ are readings scale factor.

Equations (2)-(4) can be written as,

$$
\left[\begin{array}{llll}
M_{x}^{\prime} & M_{y}^{\prime} & M_{z}^{\prime}
\end{array}\right]=\left[\begin{array}{llll}
1 & M_{x} & M_{y} & M_{z}
\end{array}\right]\left[\begin{array}{ccc}
b_{x} & b_{y} & b_{z} \\
S_{x} & 0 & 0 \\
0 & S_{y} & 0 \\
0 & 0 & S_{z}
\end{array}\right] \ldots
$$

The misalignments of magnetometer are considered as negligible, i.e., there are zero misalignments.

Equation (7) can be transformed as follows,

$$
\mathrm{H}_{1 \times 3}^{\prime}=\mathrm{H}_{1 \times 4} \mathrm{X}_{4 \times 3}
$$

Or

$\mathrm{H}_{4 \times 1}^{\mathrm{T}} \mathrm{H}_{1 \times 3}^{\prime}=\mathrm{H}_{4 \times 1}^{\mathrm{T}} \mathrm{H}_{1 \times 4} \mathrm{X}_{4 \times 3}$

Or

$\left(\mathrm{H}_{4 \times 1}^{\mathrm{T}} \mathrm{H}_{1 \times 4}\right)^{-1} \mathrm{H}_{4 \times 1}^{\mathrm{T}} \mathrm{H}_{1 \times 3}^{\prime}=\mathrm{X}_{4 \times 3}$

$\left(\mathrm{H}^{\mathrm{T}} \mathrm{H}\right)_{4 \times 4}^{-1} \mathrm{H}_{4 \times 1}^{\mathrm{T}} \mathrm{H}_{1 \times 3}^{\prime}=\mathrm{X}_{4 \times 3}$

Where, 


$$
\mathrm{H}_{1 \times 3}^{\prime}=\left[\begin{array}{lll}
M_{x}^{\prime} & M_{y}^{\prime} M_{z}^{\prime}
\end{array}\right], \mathrm{H}_{1 \times 4}=\left[\begin{array}{llll}
1 & M_{x} & M_{y} & M_{z}
\end{array}\right] \& \mathrm{X}_{4 \times 3}=\left[\begin{array}{ccc}
b_{x} & b_{y} & b_{z} \\
S_{x} & 0 & 0 \\
0 & S_{y} & 0 \\
0 & 0 & S_{z}
\end{array}\right]
$$

From Equation (8), the unknown biases and scale factors for magnetometer raw readings can be estimated and further uses for estimation of magnetometer calibrated output.

Magnetic field raw readings are stored before the magnetometer calibration. The required external heading which is used during magnetometer calibration can be estimated by using a GNSS or any reliable external heading source. The device is assumed to aligned on the platform, on which the magnetometer is installed and having zero heading misalignment during calibration. The $\mathrm{X}$-axis is towards the east direction, the $\mathrm{Y}$-axis is towards the north direction and the $\mathrm{Z}$-axis is towards the up direction for both platform and device frames.

To transform from the local level frame to the device frame of the earth magnetic field components, the following equation is devised.

$M^{b}=\mathrm{C}_{n}^{b} M^{m}$

Where,

$M^{b}$ is the earth's magnetic field components in local level frame

$M^{m}$ is the magnetic field components in device frame

$\mathrm{C}_{n}^{b}$ is the transformation Matrix

With the corresponding pitch and roll values the heading readings are used for transforming to device frame from local level East, North, and Up (ENU) frame.

\subsection{Magnetometer calibration in lab (offline calibration)}

For the land navigation applications, the magnetometer heading is utilized for optimal navigation. To use the accurate heading of the magnetometer during the integrated navigation solution, calibrated readings and compensated parameters are required. These parameters are based on magnetometer's biases, scale factors, misalignments etc. The existing calibration method is mainly based on the magnetometer's calibration in lab using different calibrations techniques. From these calibration methods, one of the most accurate and precise calibration methods is the 12 positions calibration method. In this method, magnetometer axes are place at different 12 positions and data is recorded. From this recorded data, its parameters are calculated. From the calculated parameters, the calibrated and compensated magnetometer readings are obtained which are used to find the accurate heading. For this process, reference magnetic field is also required to obtain the precise and calibrated output of the magnetometer. To obtain the reference magnetic field we use the updated version of the world's magnetic model 2020-2025.

We use the ENU frame for the magnetometer parameters calculation through the calibration. 
We calibrated the magnetometer in laboratory using 12 positions calibration method. The parameters obtained are given in the table- 1 below.

Table-1: Magnetometer calibration parameters

\begin{tabular}{|l|l|llll|}
\hline Axis/Parameters & Mx & My & Mz & \\
\hline Bias & 0.0029164 & 0.0014059 & -0.0020832 & \\
\hline Scale Factor & 0.91139 & 0.90828 & 0.91916 & \\
\hline Misalignments & -0.0030401 & 0.0050938 & 0.001792 & -0.00032331 & 0.0011975 \\
& $-6.8934 \mathrm{e}-06$ & & & & \\
\hline
\end{tabular}

The magnetometer error model equation is given below. The parameters calculated in the table1 are used in the error model equation in order to attain the calibrated readings of the magnetometer.

$$
\begin{aligned}
& M_{c x}=b_{x}+S_{x} M_{x}^{\prime}+M_{x y} * M_{y}^{\prime}+M_{x z} * M_{z}^{\prime} \\
& M_{c y}=b_{y}+S_{y} M_{y}^{\prime}+M_{y x} * M_{x}^{\prime}+M_{y z} * M_{z}^{\prime} \\
& M_{c z}=b_{z}+S_{z} M_{z}^{\prime}+M_{z x} * M_{x}^{\prime}+M_{z y} * M_{y}^{\prime}
\end{aligned}
$$

Where,

$\left[M_{c x}, M_{c y} \& M_{c z}\right]$ are magnetometer's calibrated readings

$\left[M_{x}^{\prime} M_{y}^{\prime} M_{z}^{\prime}\right]$ are magnetometer's raw readings (un-calibrated components)

$\left[b_{x}, b_{y} \& b_{z}\right]$ are biases

$\left[S_{x}, S_{y} \& S_{z}\right]$ are scale factors

$\left[M_{x y}, M_{x z}, M_{y x}, M_{y z} \& M_{z x}, M_{z y}\right]$ are misalignments

The heading can be estimated by using the calibrated horizontal readings in the following way.

$$
\text { headingc }=\arctan \cdot\left(\frac{M c y}{M c x}\right) \pm \text { Dec angle }
$$

Where,

$\left[M_{c x}, M_{c y}\right]$ are magnetometer horizontal calibrated readings.

\subsection{Magnetometer online calibration}

The main challenge of today's novel research is the online calibration of magnetometers. Since the magnetometer online heading can be used for the best navigation solution, that's why online calibration of the magnetometer is very useful to estimate the accurate heading. The raw output 
of the magnetometer was divided into sectors according to the pitch-roll sectors. The magnetometer in equations (4), (5) and (6) are used to calculate the raw output. From these equations, it is clear that there are minimum three unknowns required to estimate the raw output. By knowing the offsets, scale factors and by using the world magnetic field model 2020-2025, we can estimate the magnetometer calibrated readings. Equations (10-12) shows the error model from which the calibrated and compensated output of magnetometer can be attained. To solve these equations, a minimum of three different raw outputs of the magnetometers are required to calculate the calibrated parameters of the magnetometer for each pitch-roll sector.

\subsection{Reference Inertial Navigation System}

A high-end reference calibrated INS is used during the magnetometer's online calibration process for accurate external heading. The reference INS is precise and accurate navigation system. Sufficient data of roll and pitch sectors is required for online calibration of the magnetometer. The external heading attained by Ref. INS will be used during the magnetometer calibration. After performing magnetometer online calibration using the proposed technique, its calibrated heading can be used independently during any integrated navigation applications.

\subsection{GNSS specifications}

A NovAtel GNSS receiver and antenna were used during magnetometer's online calibration for heading and other parameters comparison. GNSS heading technology determines, where an object is pointing with respect to the true north on the horizontal plane. The NovAtel's heading solutions are suitable for many object pointing applications and the satellite communication antennas. NovAtel's GNSS technology is capable of providing decimetre-level accuracy.

Table-2: GNSS specification

\begin{tabular}{|c|c|}
\hline \multicolumn{2}{|c|}{ GNSS Specification } \\
\hline Position Accuracy & $5 \mathrm{~m}$ \\
\hline Velocity Accuracy & $0.2 \mathrm{~m} / \mathrm{sec}$ \\
\hline
\end{tabular}

\subsection{Field testing for magnetometer online calibration}

To perform online calibration of magnetometer, a field test on a system van was performed. In this field testing an AHRS comprising of magnetometer, a high-end reference Inertial Navigation System (INS) and a NovAtel GNSS was installed on a system van for field testing. Combined readings data of all systems for different pitch-roll sectors were recorded. The value of pitch-roll sectors lies in +25 degrees to -25 degrees. The field testing was executed while AHRS, INS and GNSS were in integrated mode.

\section{Field test results and discussion}

Since a combined field test of magnetometer-AHRS, a reference INS and NovAtel GNSS were conducted and data was logged for further processing. The trajectory followed during the field testing for online calibration is shown in the figure 2 below. 
Figure 2: Plot of trajectory for Magnetometer's online calibration

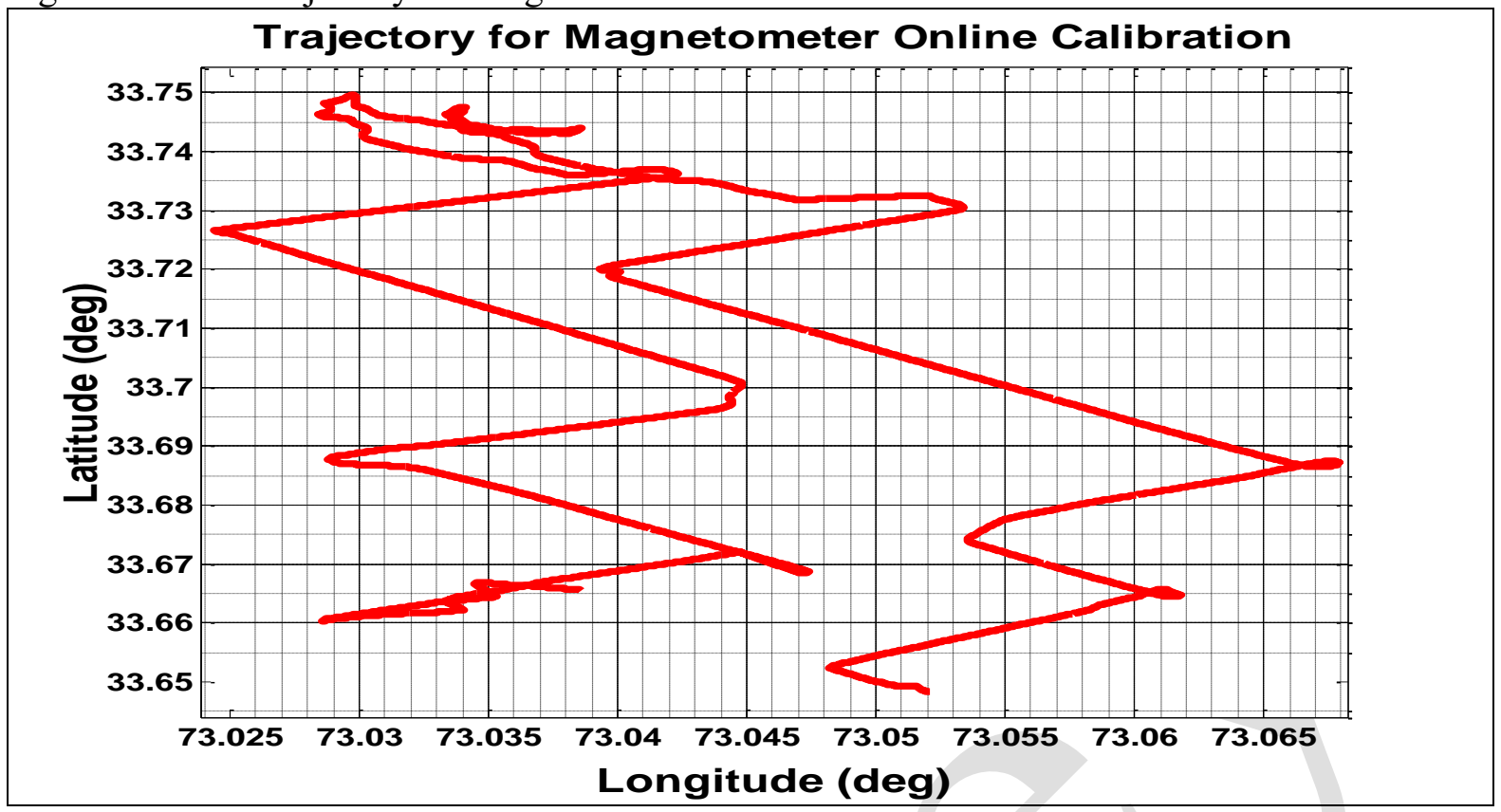

The magnetometer online calibration data was processed in figures 3-5. The comparison of magnetometer raw magnetic field components and World Magnetic Model field components is performed. This analysis describes that the magnetometer raw readings have some impurity which have to be compensated first to use it for heading estimations. The results were plotted and described as follows:

1. Plots of magnetometer $(\mathrm{Mx}, \mathrm{My}$ and $\mathrm{Mz}$ ) raw data components against World Magnetic Models magnetic field components.

Figure 3: Comparison of magnetometer raw data $\mathrm{X}$ component and world magnetic field $\mathrm{X}$ component

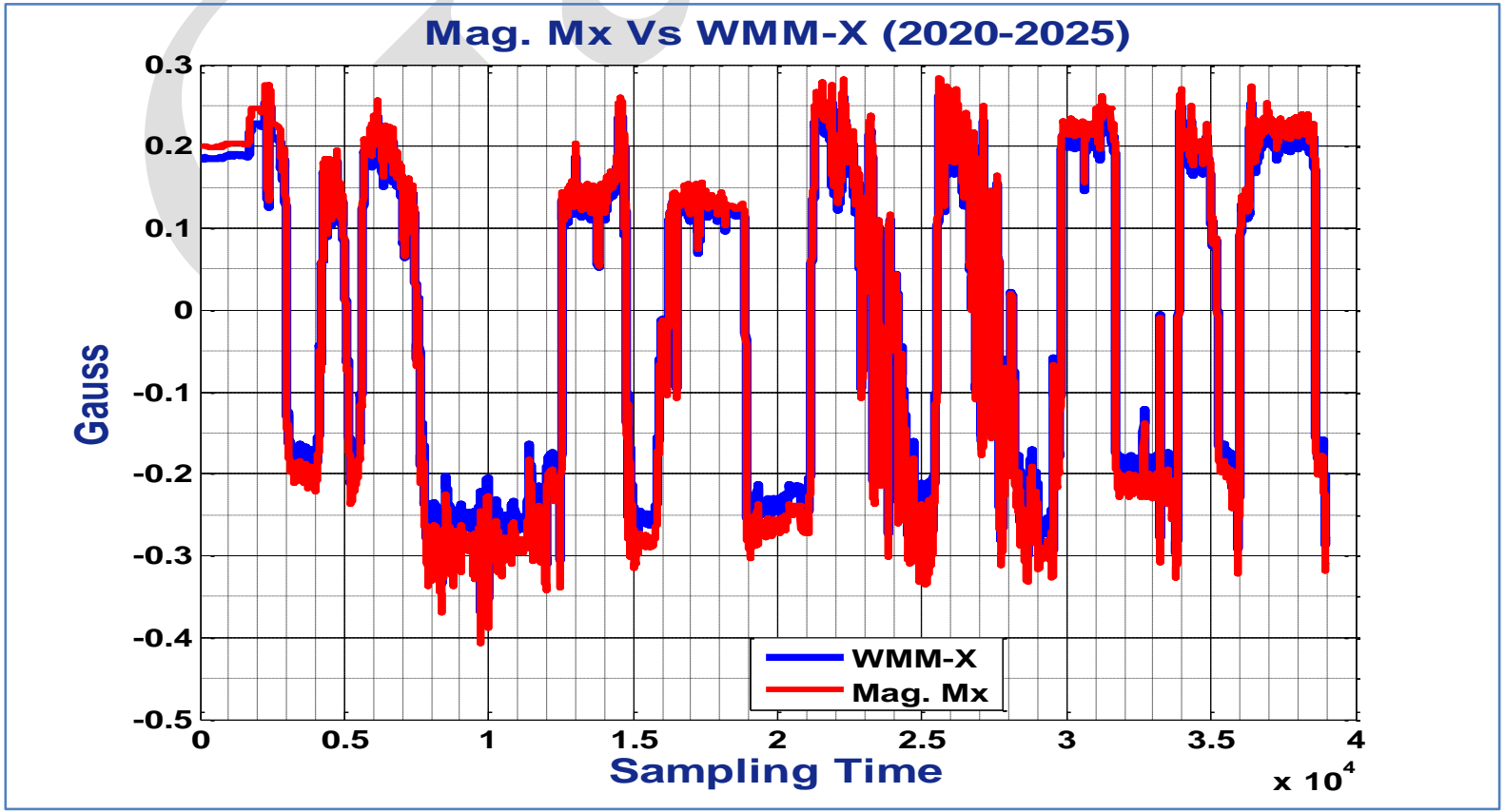


Figure 4: Comparison of magnetometer raw data $\mathrm{Y}$ component and world magnetic field $\mathrm{Y}$ component

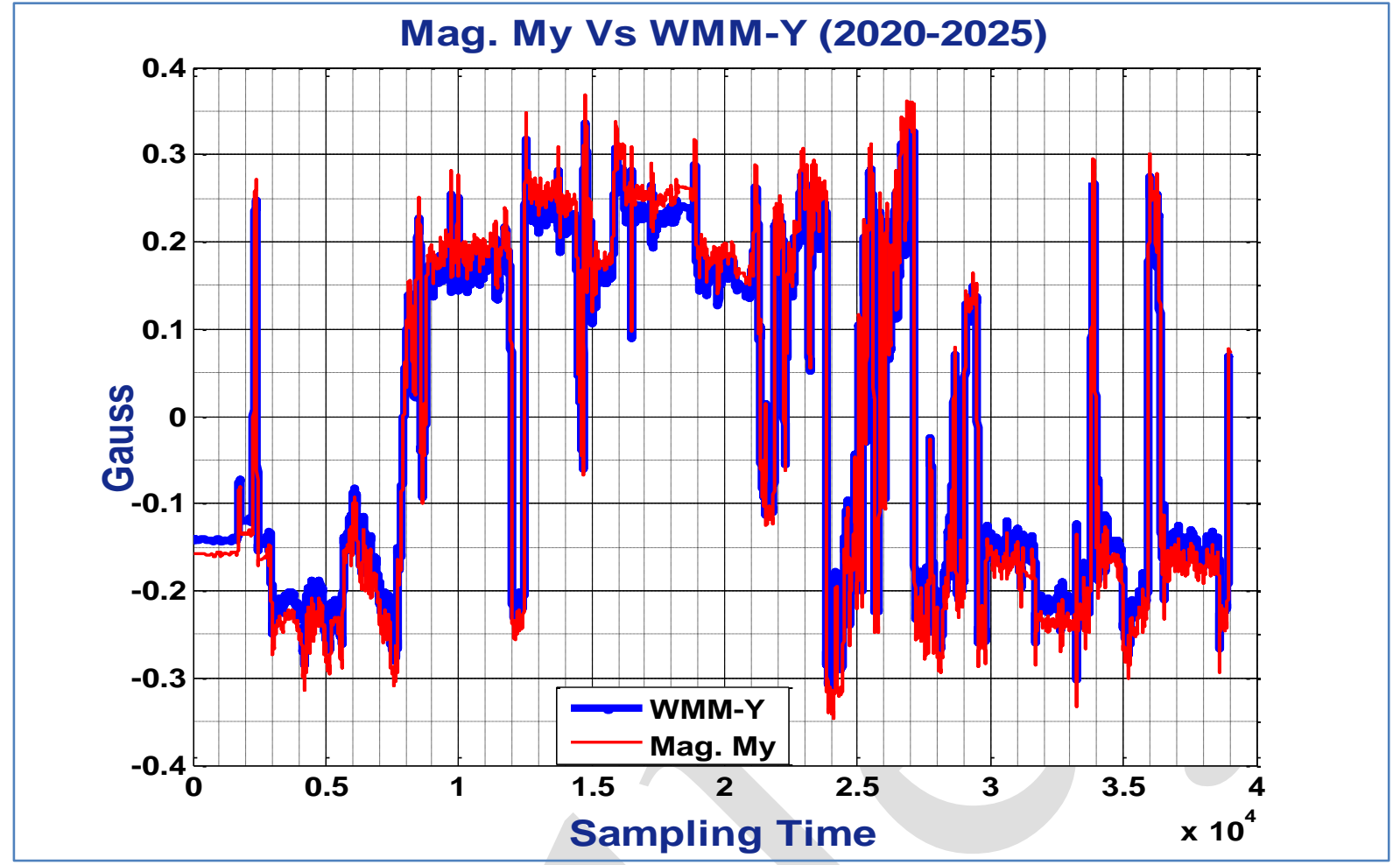

Figure 5: Comparison of magnetometer raw data $\mathrm{Z}$ component and world magnetic field $\mathrm{Z}$ component

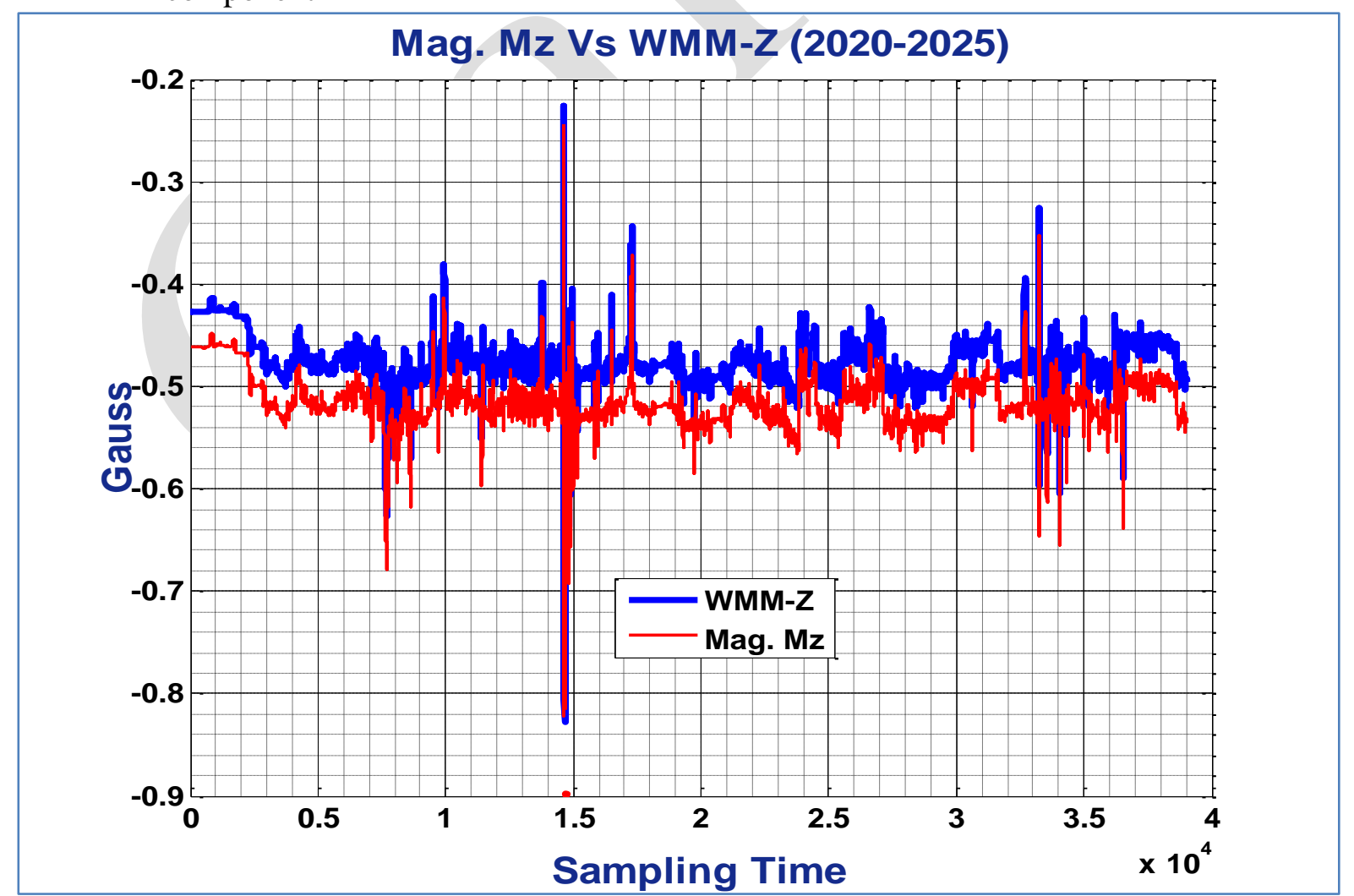


The comparison of the magnetometer components ( $\mathrm{x}, \mathrm{y}$ and $\mathrm{z}$ ) of magnetic field readings with that of earth's World Magnetic Model components (x,y and z) shows that they are not matching as shown in figures 3-5. The magnetometer raw readings have some error sources which needs to be compensated. For these compensatable parameters estimation, online calibration of magnetometer has been formed.

Since, AHRS also used in this research. The AHRS is medium accuracy navigation system. It is used with GNSS for optimal navigation results. The attitude obtained by AHRS is less acuurate as compared to the attitude of Ref. INS.

2. Plots of magnetometer heading estimated by online calibration and heading through 12 positions testing.

The data of the magnetometer's headings estimated through online calibration and offline lab calibration are plotted by using MATLAB. The plots are very identical and comparable, which means that the heading estimated through the online calibration technique is as accurate as estimated by the conventional lab calibration.

Figure 6: Comparison plots of mag. heading through online calibration (blue), mag. heading estimated through 12 positions test (brown)

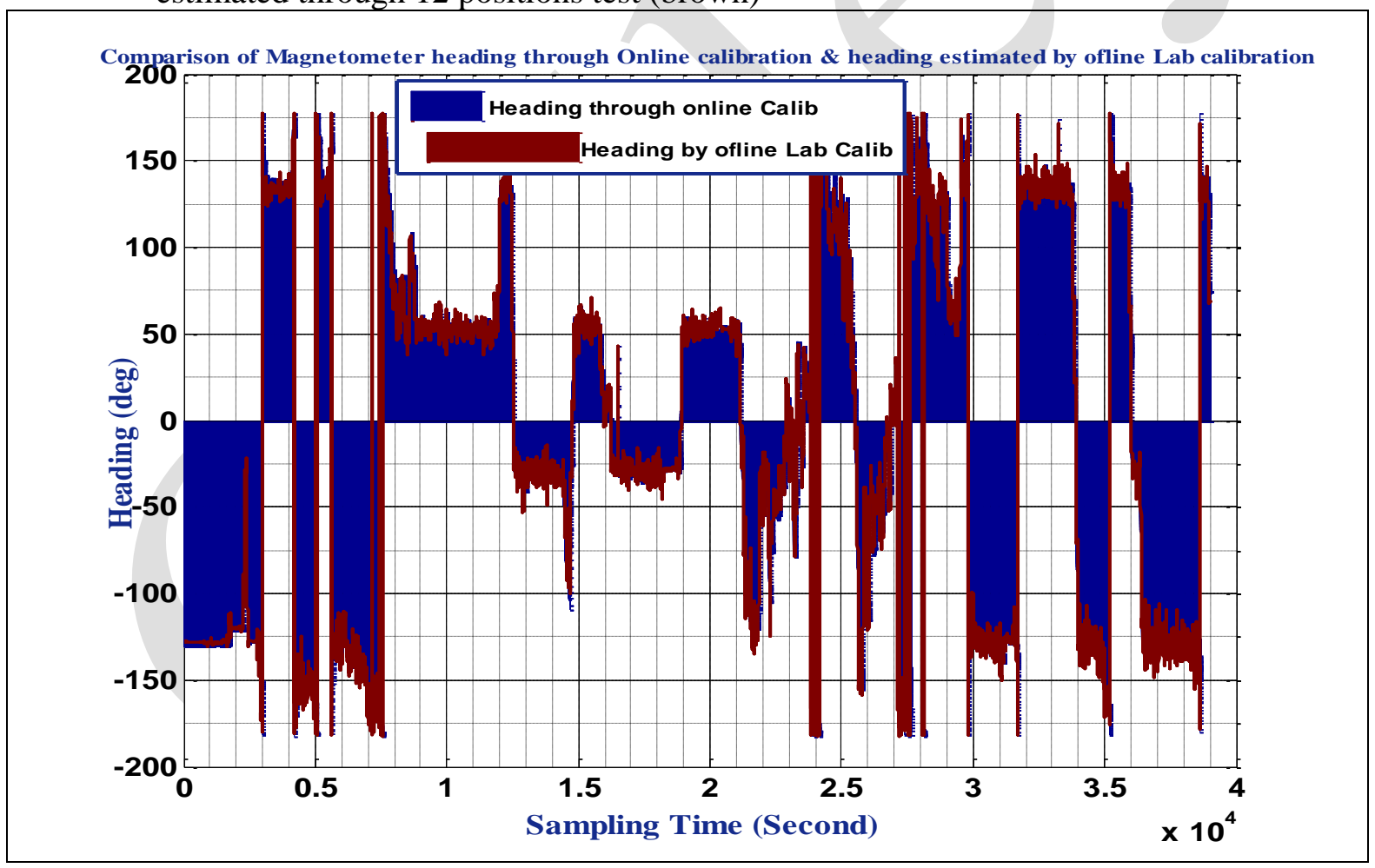

3. Plots of magnetometer heading through online calibration, heading estimated by 12 positions test and INS reference heading.

From figures 6-7, the heading through online calibration and reference INS heading are very close and almost similar. It is also clear that the magnetometer heading estimated through online calibration and through 12 positions calibration are also analogous. These are also comparable to the reference INS heading as shown in figure 7. 
Figure 7: Comparison plots of magnetometer's heading through online calibration (blue), heading from 12-positions test (green) and INS ref. heading (red)

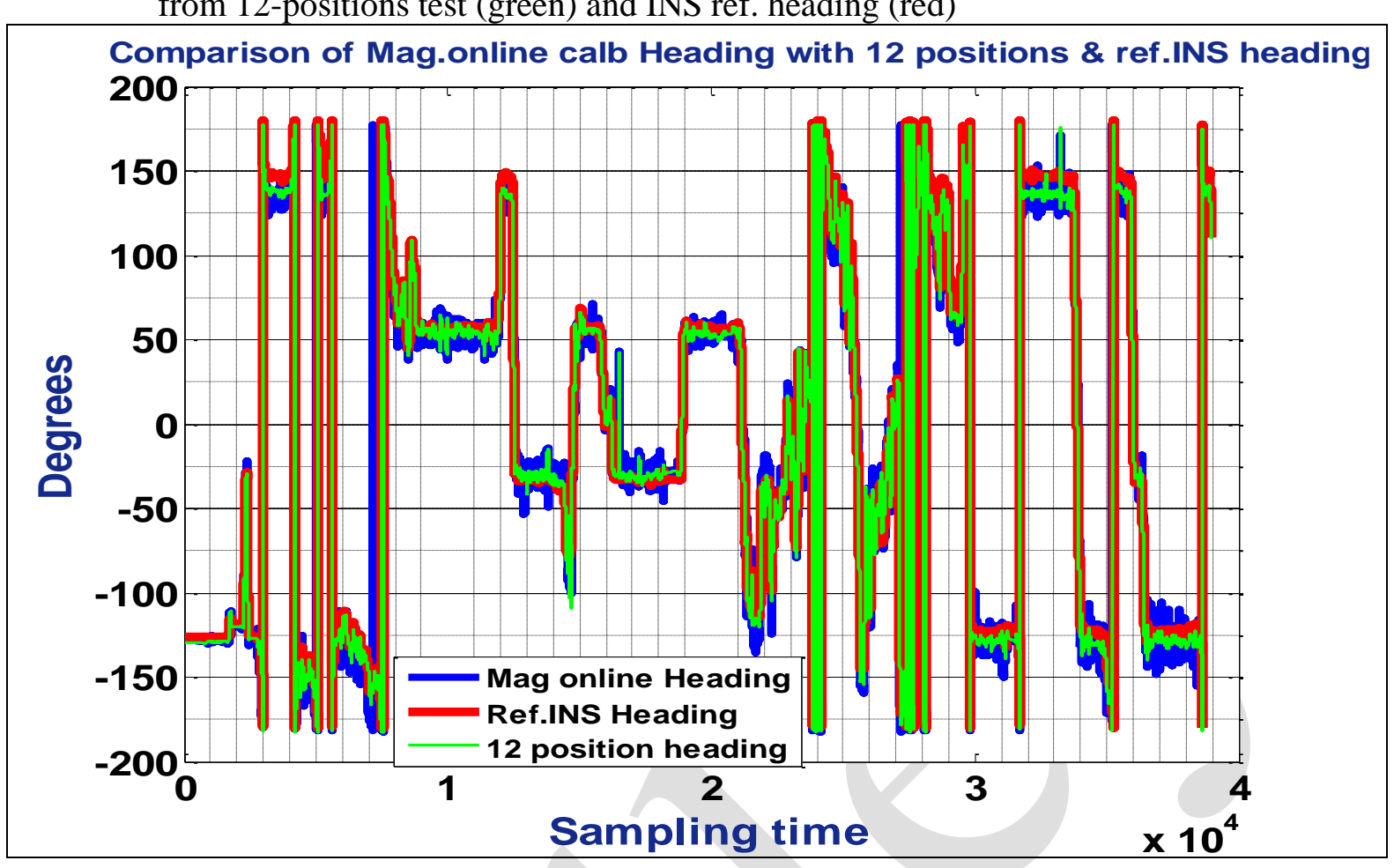

\section{Discussion}

In this paper, we used AHRS method for calibration. The attitude is obtained by IMU part and heading is obtained by the magnetometer of AHRS. Based on the analysis of the above experimental results, it can be concluded that the heading estimated through magnetometer online calibration technique is very analogous to the heading estimated by the offline lab calibration method. However, the online calibration method is faster, take less space and is more efficient. Furthermore, the magnetometer online calibration is performed autonomously and automatically. Since, the magnetometer lab based calibrations involved human interactions to perform some specific orientations. However, no human interactions were involved during the magnetometer online calibration. The heading obtained by this technique is as accurate as obtained by the conventional lab calibration. Therefore, magnetometer can be integrated with navigation system to provide accurate heading information during land navigation.

\section{Conclusion}

The attitude obtained by AHRS was analysed and compared with the attitude of reference INS. The AHRS attitude was found noisier as compared to the reference INS attitude. Therefore, by using the Kalman filter AHRS attitude can be refined for usage of navigation applications. Also, by the integration of AHRS and GNSS, we can achieve good navigation results for land navigation applications. Different pitch-roll sectors were processed by using reference INS and magnetometer data to calculate the accurate biases, scale factors and misalignments for these pitch-roll sectors. Then, an accurate heading was estimated by using the calibrated readings. Therefore, the magnetometer's calibrated heading estimated by the proposed method is comparable to the heading obtained by the conventional lab calibration methods. The magnetometer's heading and reference INS heading are analogous. Therefore, magnetometer 
heading information can independently be used for land navigation. Also, during integrated navigation applications, when GNSS is not available then magnetometer heading can be used to accomplish the land navigation mission. Overall, the designed system can intensify the integrated navigation solution for land navigation applications.

\section{Acknowledgement}

The authors would like to thank the center of excellence of national engineering and scientific commission and institute of space technology for facilitating to conduct the research work.

\section{References}

Aggarwal, P., Syed, Z., Niu, X., \& El-Sheimy, N. (2008). A standard testing and calibration procedure for low cost MEMS inertial sensors and units. The Journal of Navigation, 61(2), 323-336. https://doi.org/10.1017/S0373463307004560

D’Amato, E., De Capua, C., Filianoti, P. F., Gurnari, L., Nardi, V. A., Notaro, I., \& Scordamaglia, V. (2021, October). UKF-based fault detection and isolation algorithm for IMU sensors of Unmanned Underwater Vehicles. In 2021 International Workshop on Metrology for the Sea; Learning to Measure Sea Health Parameters (MetroSea) (pp. 371-376).

IEEE. https://doi.org/10.1109/MetroSea52177.2021.9611599

Caruso, M. J. (1997). Applications of magnetoresistive sensors in navigation systems (No. 970602). SAE Technical Paper. https://doi.org/10.4271/970602

Caruso, M. J. (2000, March). Applications of magnetic sensors for low cost compass systems. In IEEE 2000. Position location and navigation symposium (Cat. No. 00CH37062) (pp. 177-184). IEEE. https://doi.org/10.1109/PLANS.2000.838300

Fan, C., Hu, X., He, X., Tang, K., \& Luo, B. (2014). Observability analysis of a MEMS INS/GPS integration system with gyroscope G-sensitivity errors. Sensors, 14(9), 16003-16016. https://doi.org/10.3390/s140916003

Chiang, K. W., Noureldin, A., \& El-Sheimy, N. (2003). Multisensor integration using neuron computing for land-vehicle navigation. GPS Solutions, 6(4), 209-218. https://link.springer.com/content/pdf/10.1007/s10291-002-0024-4.pdf

Gebre-Egziabher, D., Elkaim, G. H., David Powell, J., \& Parkinson, B. W. (2006). Calibration of strapdown magnetometers in magnetic field domain. Journal of Aerospace Engineering, 19(2), 87-102. https://ascelibrary.org/doi/abs/10.1061/(ASCE)0893$\underline{1321(2006) 19: 2(87)}$

Groves, P. D. (2013). The PNT boom: Future trends in integrated navigation. Inside GNSs, 8(2), 44-49. https://discovery.ucl.ac.uk/id/eprint/1394442/

Ilyas, M., Yang, Y., Qian, Q. S., \& Zhang, R. (2013, May). Low-cost IMU/odometer/GPS integrated navigation aided with two antennae heading measurement for land vehicle application. In 2013 25th Chinese Control and Decision Conference (CCDC) (pp. 4521-4526). IEEE. https://doi.org/10.1109/CCDC.2013.6561750

Javed, M. A., \& Tahir, M. (2021). A gyroless attitude estimation for ground vehicle under severe dynamic conditions. IEEE Transactions on Intelligent Transportation Systems. https://doi.org/10.1109/TITS.2021.3125712

Kaplan, E. D., \& Hegarty, C. J. (1996). Understanding GPS, principles and applications. Artech House. 
Kok, M., \& Schön, T. B. (2016). Magnetometer calibration using inertial sensors. IEEE Sensors Journal, 16(14), 5679-5689.

Lim, J., Yoo, W. J., Kim, L. W., Lee, Y. D., \& Lee, H. K. (2018). Augmentation of GNSS by low-cost MEMS IMU, OBD-II, and digital altimeter for improved positioning in urban area. Sensors, 18(11), 3830. https://doi.org/10.3390/s18113830

Lee, J., Sung, C. K., Oh, J., Han, K., Lee, S., \& Yu, M. J. (2020). A pragmatic approach to the design of advanced precision terrain-aided navigation for UAVs and Its verification. Remote Sensing, 12(9), 1396. https://doi.org/10.3390/rs12091396

Mansoor, S., Bhatti, U. I., Bhatti, A. I., \& Ali, S. M. D. (2019). Improved attitude determination by compensation of gyroscopic drift by use of accelerometers and magnetometers. Measurement, 131,

582-589. https://doi.org/10.1016/j.measurement.2018.08.067

Noureldin, A., Karamat, T. B., \& Georgy, J. (2013). Fundamentals of inertial navigation, satellite-based positioning and their integration. https://link.springer.com/content/pdf/bfm\%253A978-3-642-30466-8\%252F1.pdf

Ozevin, D. (2014). Micro-electro-mechanical-systems (MEMS) for assessing and monitoring civil infrastructures. In Sensor Technologies for Civil Infrastructures (pp. 265-302e). Woodhead Publishing. https://doi.org/10.1533/9780857099136.265

Rabiain, A. H., Kealy, A., \& Morelande, M. (2013). Tightly coupled MEMS based INS/GNSS performance evaluation during extended GNSS outages. Journal of Applied Geodesy, 7(4), 291-298. https://www.degruyter.com/document/doi/10.1515/jag2013-0056/html

Skog, I., \& Händel, P. (2006, September). Calibration of a MEMS Inertial Measurement Unit. In XVII IMEKO World Congress (pp. 1-6). https://citeseerX.ist.psu.edu/viewdoc/download?doi=10.1.1.65.5896\&rep=rep1\&typ $\underline{\mathrm{e}=\mathrm{pdf}}$

Titterton, D., Weston, J. L., \& Weston, J. (2004). Strapdown inertial navigation technology (Vol. 17). IET.

Wahdan, A., Georgy, J., \& Noureldin, A. (2014). Three-dimensional magnetometer calibration with small space coverage for pedestrians. IEEE Sensors Journal, 15(1), 598-609. https://doi.org/10.1109/JSEN.2014.2348552

Won, D., Ahn, J., Sung, S., Heo, M., Im, S. H., \& Lee, Y. J. (2015). Performance improvement of inertial navigation system by using magnetometer with vehicle dynamic constraints. Journal of Sensors, 2015. https://www.hindawi.com/journals/js/2015/435062/

Yang, L., Li, Y., Wu, Y., \& Rizos, C. (2014). An enhanced MEMS-INS/GNSS integrated system with fault detection and exclusion capability for land vehicle navigation in urban areas. Gps Solutions, 18(4), 593-603. https://link.springer.com/content/pdf/10.1007/s10291-013-0357-1.pdf

Yongjian, Z., Lin, W., Guo, W., Chunfeng, G., \& Hui L., (2021). Integrated navigation method of RLG INS/GNSS polar region. Infrared and Laser Engineering, 50(S2), 20210066. http://dx.doi.org/10.3788/IRLA20210066

Zhou, Z., Lyu, P., Lai, J., Zhu, X., \& Huang, K. (2022). Multi-vehicle collaborative navigation Method based on datalink relative distance in GNSS denied environment. In Advances in Guidance, Navigation and Control (pp. 3875-3888). Springer, Singapore. https://link.springer.com/chapter/10.1007/978-981-15-8155-7_324 